\title{
A Characterization of Decidable Locally Finite Varieties
}

\author{
Ralph McKenzie Matthew A. Valeriote*
}

August 1, 1989

\begin{abstract}
We describe the structure of those locally finite varieties whose first order theory is decidable. A variety is a class of universal algebras defined by a set of equations. Such a class is said to be locally finite if every finitely generated member of the class is finite. It turns out that in order for such a variety to have a decidable theory it must decompose into the varietal product of three special kinds of varieties; a strongly Abelian variety; an affine variety; and a discriminator variety.
\end{abstract}

\section{Introduction}

Since the 1930's, when precise notions of algorithm and decidability were introduced, the decidability or undecidability of many familiar varieties has been determined. For example, Tarski [21] proved that the theory of Boolean algebras is decidable and in a series of papers $[20,7,25]$ it was shown that a variety of groups is decidable if and only if it contains no non-Abelian group.

In 1986 the authors, building on the work of Burris and McKenzie [3], Zamyatin $[23,24,25]$ and others were able to give an algebraic description of the decidable locally finite varieties. In this paper we describe this structure and give a rough outline of the proof. Complete details can be found in [17]. One of the principle tools used in this work is the recently discovered theory

*AMS Subject Classification, Primary: 03B25 Secondary: 08A05

The first author was supported by the U. S. NSF and the second by the NSERC of Canada. 
called tame congruence theory. This theory was developed in the early 1980's by McKenzie and his student David Hobby. We will present a small fragment of this theory in section 3. The reader can consult [9] for more details.

By an algebra we mean simply any structure $\left\langle A, f_{i}(i \in I)\right\rangle$ consisting of a nonvoid set $A$ and a system of finitary operations $f_{i}$ over $A$. A variety, or equational class, is a class of similar algebras defined by some set of equations. A variety is called locally finite if every one of its finitely generated algebras is finite.

A variety $\mathcal{V}$ is called decidable if and only if its first order theory is a recursive set of sentences. This means that there is an algorithm which will determine, for any given sentence $\varphi$ in the language of $\mathcal{V}$, whether $\varphi$ is true for all members of $\mathcal{V}$.

Usually, to establish the undecidability of a class of structures, another class known to be undecidable is semantically embedded into the first class. The class of finite graphs, $\mathcal{G}_{\text {fin }}$, was shown to be undecidable by Lavrov in [13]. It turns out that if a locally finite variety fails to have the structure described in Theorem 2.14 then it allows a semantic embedding of this class $\mathcal{G}_{\text {fin }}$ and hence is undecidable.

\section{Special Kinds of Varieties}

We introduce the families of Abelian varieties, strongly Abelian varieties, affine varieties, and discriminator varieties. These are the varieties that are needed in our description of the locally finite decidable varieties.

Given a set $A$ and natural numbers $n>0$ and $i<n$, we can define the projection function $p_{i}^{n}: A^{n} \rightarrow A$ by

$$
p_{i}^{n}\left(x_{0}, \ldots, x_{n-1}\right)=x_{i} \text { for all } x_{0}, \ldots, x_{n-1} \in A .
$$

A clone on a set $A$ is a set of finitary operations on $A$ that contains the projections $p_{i}^{n}$ for all $0 \leq i<n<\omega$, and is closed under composition of operations. By a polynomial clone on $A$, we mean any clone on $A$ that also contains all of the constant 0-ary operations on $A$. (A general clone is not required by our definition to contain any 0 -ary operations.)

Associated with any algebra $\mathbf{A}=\left\langle A, f^{\mathbf{A}}(f \in \Phi)\right\rangle$ are two important clones. The clone of term operations of $\mathbf{A}$, denoted Clo $\mathbf{A}$, is the clone on $A$ generated by the set $\left\{f^{\mathbf{A}}: f \in \Phi\right\}$ of basic operations of $\mathbf{A}$. An 
$n$-ary operation $f$ on $A$ belongs to Clo $\mathbf{A}$ iff there exists a term $t$ in the language $\mathbf{L}$ such that $f=t^{\mathbf{A}}$-i.e., $f$ is the operation induced by the term $t$. The clone of polynomial operations of $\mathbf{A}$, denoted $\mathrm{Pol} \mathbf{A}$, is the clone on $A$ generated by the basic operations of $\mathbf{A}$ along with all of the constant 0 -ary operations on $A$. We write $\mathrm{Clo}_{\mathrm{n}} \mathbf{A}$ for the set of all $n$-ary members of Clo $\mathbf{A}$ ( $n$-ary term operations of $\mathbf{A}$ ); and, similarly, we write $\operatorname{Pol}_{n} \mathbf{A}$ for the set of all $n$-ary members of Pol $\mathbf{A}$ ( $n$-ary polynomial operations of $\mathbf{A}$ ). It follows that if $p \in \operatorname{Pol}_{\mathrm{n}} \mathbf{A}$, then for some $m \in \omega$ and $a_{0}, \ldots, a_{m-1} \in A$ and for some $f \in \mathrm{Clo}_{\mathrm{m}+\mathrm{n}} \mathbf{A}$, we have $p(\bar{x})=f(\bar{x}, \bar{a})$ for all $\bar{x} \in A^{n}$ (where $\left.\bar{a}=\left\langle a_{0}, \ldots, a_{m-1}\right\rangle\right)$.

Definition 2.1 Algebras A and B are said to be polynomially equivalent if they have the same universe and precisely the same polynomial operations, i.e., if $\mathrm{Pol} \mathbf{A}=\mathrm{Pol} \mathbf{B}$.

The concept of an Abelian algebra was introduced in the 1970's and has played an important role in the study of both congruence modular varieties and locally finite varieties.

Definition 2.2 Let $\alpha, \beta, \gamma \in$ Con A. We write $\mathrm{C}(\alpha, \beta ; \gamma)$, and say that $\alpha$ centralizes $\beta$ modulo $\gamma$, provided that the following condition holds:

For every $n \geq 1$, for every $f \in \mathrm{Clo}_{\mathrm{n}+1} \mathbf{A}$, and for all $\langle a, b\rangle \in \alpha$ and $\left\langle c_{1}, d_{1}\right\rangle, \ldots,\left\langle c_{n}, d_{n}\right\rangle \in \beta$ we have

$$
f(a, \bar{c}) \equiv_{\gamma} f(a, \bar{d}) \leftrightarrow f(b, \bar{c}) \equiv_{\gamma} f(b, \bar{d}) .
$$

Definition 2.3 Let $\mathbf{A}$ be any algebra. The center of $\mathbf{A}$ is the binary relation $Z(\mathbf{A})$ defined by $\langle x, y\rangle \in Z(\mathbf{A}) \longleftrightarrow$

for all $n \geq 1$, for all $f \in \mathrm{Clo}_{\mathrm{n}+1} \mathbf{A}$, and for all $c_{1}, d_{1}, \ldots, c_{n}, d_{n} \in A$

$$
f(x, \bar{c})=f(x, \bar{d}) \leftrightarrow f(y, \bar{c})=f(y, \bar{d}) .
$$

It is a congruence on $\mathbf{A}$. The algebra $\mathbf{A}$ is called Abelian iff $Z(\mathbf{A})=1_{A}$, and called centerless iff $Z(\mathbf{A})=0_{A}$.

Definition 2.4 Let $\mathbf{A}$ be any algebra, $\alpha, \beta, \gamma, \delta$ be congruences on $\mathbf{A}$. 
(1) If $\alpha \leq \beta$, we say that $\beta$ is Abelian over $\alpha$ iff $\mathrm{C}(\beta, \beta ; \alpha)$.

(2) If $\delta \leq \gamma$, we say that $\gamma$ is solvable over $\delta$ iff there exists a finite chain of congruences $\alpha_{0}=\delta \leq \alpha_{1} \leq \cdots \leq \alpha_{n}=\gamma$ with $\alpha_{i+1}$ Abelian over $\alpha_{i}$ for all $i<n$.

(3) If $\delta \leq \gamma$, we say that $\gamma$ is locally solvable over $\delta$ iff for every finitely generated subalgebra $\mathbf{B} \leq \mathbf{A}$ and for the restricted congruences $\left.\delta\right|_{B}$ and $\left.\gamma\right|_{B}$ of $\mathbf{B}$, we have that $\left.\gamma\right|_{B}$ is solvable over $\left.\delta\right|_{B}$.

(4) We say that $\mathbf{A}$ is solvable iff $1_{A}$ is solvable over $0_{A}$; locally solvable iff $1_{A}$ is locally solvable over $0_{A}$.

We now strengthen the Abelian property in two mutually incompatible ways: to strongly Abelian algebras and to affine algebras.

Definition 2.5 Let $\alpha \leq \beta$ be congruences of an algebra $\mathbf{A}$. We say that $\beta$ is strongly Abelian over $\alpha$ iff for all $n \geq 1$, for all $f \in \mathrm{Clo}_{\mathrm{n}+1} \mathbf{A}$, and for all $a \equiv b \equiv c(\bmod \beta)$ and $\left\langle u_{1}, v_{1}\right\rangle, \ldots,\left\langle u_{n}, v_{n}\right\rangle \in \beta$ we have

$$
f(a, \bar{u}) \equiv_{\alpha} f(b, \bar{v}) \rightarrow f(c, \bar{u}) \equiv_{\alpha} f(c, \bar{v}) .
$$

We say that $\mathbf{A}$ is strongly Abelian iff $1_{A}$ is strongly Abelian over $0_{A}$.

Definition 2.6 Let $\delta \leq \gamma$ be congruences of an algebra $\mathbf{A}$.

(1) We say that $\gamma$ is strongly solvable over $\delta$ iff there exists a finite chain of congruences $\alpha_{0}=\delta \leq \alpha_{1} \leq \cdots \leq \alpha_{n}=\gamma$ such that $\alpha_{i+1}$ is strongly Abelian over $\alpha_{i}$ for all $i<n$.

(2) We say that $\gamma$ is locally strongly solvable over $\delta$ iff for every finitely generated subalgebra $\mathbf{B} \leq \mathbf{A}$ and for the restricted congruences $\left.\delta\right|_{B}$ and $\left.\gamma\right|_{B}$ of the algebra $\mathbf{B}$, we have that $\left.\gamma\right|_{B}$ is strongly solvable over $\left.\delta\right|_{B}$.

(3) The algebra $\mathbf{A}$ is said to be strongly solvable iff $1_{A}$ is strongly solvable over $0_{A}$; and is said to be locally strongly solvable iff $1_{A}$ is locally strongly solvable over $0_{A}$.

The proof of the following theorem can be found in [9]. 
THEOREM 2.7 For every locally finite variety $\mathcal{V}$, the class of all locally solvable algebras in $\mathcal{V}$, and the class of all locally strongly solvable algebras in $\mathcal{V}$, are varieties.

Two equivalence relations $\alpha$ and $\beta$ on a set $A$ are said to permute iff whenever $a \alpha b \beta c$ there exists some element $d$ such that $a \beta d \alpha c$. A. I. Maltsev [14] proved that a variety $\mathcal{V}$ has the property that every two congruences on any algebra in $\mathcal{V}$ permute iff there exists a term $t(x, y, z)$ in the language of $\mathcal{V}$ for which the equations $t(x, y, y) \approx x$ and $t(x, x, y) \approx y$ are valid in $\mathcal{V}$. When such a term exists, we say that $\mathcal{V}$ is Maltsev. An operation on a set $A$ that obeys these two equations on $A$ is called a Maltsev operation; also, an algebra having a term operation that obeys these equations is called a Maltsev algebra. A useful corollary of Maltsev's result is that a variety $\mathcal{V}$ is Maltsev if and only if the free algebra $\mathbf{F}_{\mathcal{V}}(3)$ has permuting congruences.

Definition 2.8 An algebra $\mathbf{A}$ is called affine iff $\mathbf{A}$ is polynomially equivalent with an algebra $\mathbf{M}$ that is a module over a ring.

\section{Definition 2.9}

(1) If (P) is any one of the properties "Abelian", "strongly Abelian", "affine", "locally solvable", "locally strongly solvable" defined above, we say that a variety is $(\mathrm{P})$ iff every algebra in the variety is $(\mathrm{P})$.

(2) A variety $\mathcal{V}$ is said to be congruence-modular (or -distributive) iff the congruence lattice of each algebra in $\mathcal{V}$ is a modular (or distributive) lattice.

The basic results concerning affine algebras and affine varieties are fully detailed in Freese, McKenzie [8]. We describe these results here without giving proofs. Note that if $\mathcal{V}$ is affine, then, since the free algebra on three generators in $\mathcal{V}$ has a Maltsev term operation, it follows that $\mathcal{V}$ is Maltsev.

THEOREM 2.10 An algebra $\mathbf{A}$ is affine if and only if it satisfies one of these conditions (which are equivalent).

(i) $\mathrm{A}$ is Abelian and possesses a polynomial operation $p(x, y, z)$ obeying the equations $p(x, y, y) \approx x$ and $p(x, x, y) \approx y$. 
(ii) A possesses a term operation $p(x, y, z)$ obeying the above equations and such that for every basic operation $f$ of $\mathbf{A}$, the equation

$$
p(f(\bar{x}), f(\bar{y}), f(\bar{z})) \approx f\left(p\left(x_{0}, y_{0}, z_{0}\right), \ldots, p\left(x_{n-1}, y_{n-1}, z_{n-1}\right)\right)
$$

is valid in $\mathbf{A}$ (if $f$ is $n$-ary).

THEOREM 2.11 $A$ variety $\mathcal{V}$ is affine if and only if it is congruencemodular and Abelian. If $\mathcal{V}$ is affine then it is in fact Maltsev; and there exists a term $t(x, y, z)$ in the language of $\mathcal{V}$ and a ring $\mathbf{R}$ with unit such that every algebra in $\mathcal{V}$ is polynomially equivalent with a unitary left $\mathbf{R}$-module in which $x-y+z=t(x, y, z)$, and every unitary left $\mathbf{R}$-module is polynomially equivalent with an algebra in $\mathcal{V}$.

The concept of a discriminator variety is in many respects the polar opposite of that of an Abelian variety. On any set $U$ we can define an operation $t_{U}(x, y, z)$ by stipulating that $t_{U}(x, y, z)$ is $z$ if $x=y$, and is $x$ if $x \neq y$. This operation $t_{U}$ is called the ternary discriminator on $U$.

Definition 2.12 A variety $\mathcal{V}$ is called a discriminator variety iff there exists a term $t(x, y, z)$ in the language of $\mathcal{V}$ such that $\mathcal{V}=\mathrm{V}(\mathcal{S})$ where $\mathcal{S}$ is the class of all $\mathbf{A} \in \mathcal{V}$ such that $t^{\mathbf{A}}=t_{A}$ (i.e., the term $t$ defines the discriminator on the universe of $\mathbf{A}$ ). Such a term $t$ is called a discriminator term for $\mathcal{V}$.

There is a very nice structure theory for discriminator varieties, the details of which can be found in Burris, Sankappanavar [5]. Several important facts about these varieties are given below without proof. An algebra $\mathbf{A}$ is called hereditarily simple iff $|A|>1$ and every subalgebra $\mathbf{B} \leq \mathbf{A}$ with more than one element is simple. A variety $\mathcal{V}$ is called arithmetical iff $\mathcal{V}$ is congruence-distributive and Maltsev.

THEOREM 2.13 Let $\mathcal{V}$ be a discriminator variety with discriminator term $t$. Then $\mathcal{V}$ is an arithmetical variety. The equations

$$
t(x, y, y) \approx t(x, y, x) \approx t(y, y, x) \approx x
$$

are valid in $\mathcal{V}$. Every algebra in $\mathcal{V}$ is centerless; and every finite algebra in $\mathcal{V}$ is isomorphic to a direct product of simple algebras. The following are equivalent for every $\mathbf{A} \in \mathcal{V}$ : $\mathbf{A}$ is subdirectly irreducible; $\mathbf{A}$ is hereditarily simple; $t^{\mathbf{A}}=t_{A}$. 
One last concept is needed before we can state our result. Varieties $\mathcal{V}_{1}, \ldots, \mathcal{V}_{n}$ in the same language $\mathrm{L}$ are called independent iff there exists an L-term $t\left(x_{1}, \ldots, x_{n}\right)$ such that $\mathcal{V}_{i}=t \approx x_{i}$ for $i=1, \ldots, n$. If $\mathcal{V}_{1}, \ldots, \mathcal{V}_{n}$ are independent, then every algebra $\mathrm{A}$ in $\mathcal{V}=\mathcal{V}_{1} \vee \cdots \vee \mathcal{V}_{n}$ is isomorphic to a product $\mathbf{A}_{1} \times \cdots \times \mathbf{A}_{n}$ with $\mathbf{A}_{1} \in \mathcal{V}_{1}, \ldots, \mathbf{A}_{n} \in \mathcal{V}_{n}$ and the algebras $\mathbf{A}_{i}$ are determined up to isomorphism. In this case, we write $\mathcal{V}_{1} \otimes \cdots \otimes \mathcal{V}_{n}$ for the join variety $\mathcal{V}=\mathcal{V}_{1} \vee \cdots \vee \mathcal{V}_{n}$, and say that $\mathcal{V}$ is the product of its subvarieties $\mathcal{V}_{1}, \ldots, \mathcal{V}_{n}$

THEOREM 2.14 Let $\mathcal{V}$ be any decidable locally finite variety. There exists a strongly Abelian variety $\mathcal{V}_{1}$, an affine variety $\mathcal{V}_{2}$, and a discriminator variety $\mathcal{V}_{3}$ such that

$$
\mathcal{V}=\mathcal{V}_{1} \otimes \mathcal{V}_{2} \otimes \mathcal{V}_{3}
$$

These three subvarieties of $\mathcal{V}$ are uniquely determined and are all decidable.

\section{Tame Congruence Theory}

One of the key steps in the development of tame congruence theory was the realization that locally the behaviour of finite algebras is quite limited. This is made precise in the following definitions and theorems. The reader may wish to refer to [9] for further details and proofs. (For the basic theory of universal algebra, consult [5] or [16].)

Definition 3.1 Let $\mathbf{A}$ be a finite algebra and let $\alpha$ and $\beta$ be congruences of A.

(1) We say that a function $f: A \rightarrow A$ collapses $\beta$ into $\alpha$ and write $f(\beta) \subset \alpha$ if $\langle f(a), f(b)\rangle \in \alpha$ for all $\langle a, b\rangle \in \beta$.

(2) By a congruence quotient of $\mathbf{A}$ we mean a pair $\langle\alpha, \beta\rangle$ of congruences of $\mathbf{A}$ such that $\alpha<\beta$. A congruence quotient $\langle\alpha, \beta\rangle$ of $\mathbf{A}$ is called a prime quotient iff $\beta$ covers $\alpha$ in Con $\mathbf{A}$ (the congruence lattice of A). The relation of covering between two elements of Con $\mathbf{A}$ is written $\alpha \prec \beta$.

(3) Let $\langle\alpha, \beta\rangle$ be a congruence quotient of $\mathbf{A}$ and let

$$
U_{\mathbf{A}}(\alpha, \beta)=\left\{f(A): f \in \mathrm{Pol}_{1} \mathbf{A} \text { and } f(\beta) \not \subset \alpha\right\}
$$


and $M_{\mathbf{A}}(\alpha, \beta)$ be the set of all minimal members of $U_{\mathbf{A}}(\alpha, \beta)$ relative to the ordering of inclusion. A member of $M_{\mathbf{A}}(\alpha, \beta)$ is called an $\langle\alpha, \beta\rangle$ minimal set of $\mathbf{A}$.

Definition 3.2 Let $\mathbf{A}$ be a finite algebra and suppose that $\alpha \prec \beta \in$ Con $\mathbf{A}$. By an $\langle\alpha, \beta\rangle$-trace in $\mathbf{A}$ we mean any set $N \subset A$ such that for some $U \in$ $M_{\mathbf{A}}(\alpha, \beta), N \subset U$ and $N$ is of the form $(x / \beta) \cap U$ for some $x \in U$ such that $(x / \alpha) \cap U \neq(x / \beta) \cap U$. The body and the tail of an $\langle\alpha, \beta\rangle$-minimal set $U$ with respect to $\langle\alpha, \beta\rangle$ are defined by

$$
\begin{gathered}
\text { body }=\bigcup\{\langle\alpha, \beta\rangle \text {-traces contained in } U\}, \\
\text { tail }=U-\text { body. }
\end{gathered}
$$

For $U$ a nonvoid subset of an algebra $\mathbf{A}$, we let $\left.(\mathrm{Pol} \mathbf{A})\right|_{U}$ denote the set of all $\left.f\right|_{U}$ where $f \in \operatorname{Pol} \mathbf{A}$ and $U$ is closed under $f$. The (non-indexed) algebra $\left.\mathbf{A}\right|_{U}$ having universe $U$ and fundamental operations $\left.(\mathrm{Pol} \mathbf{A})\right|_{U}$ is called the algebra induced by $\mathbf{A}$ on $U$.

In tame congruence theory we focus on the algebras induced on the minimal sets, bodies and traces of finite algebras. As the next theorem demonstrates, there are few possibilities for the algebraic structure induced on a trace by a finite algebra.

THEOREM 3.3 Let $\mathbf{A}$ be a finite algebra and let $\langle\alpha, \beta\rangle$ be a prime quotient of $\mathbf{A}$. If $N_{1}$ and $N_{2}$ are $\langle\alpha, \beta\rangle$-traces then $\left.\alpha\right|_{N_{i}}$ is a congruence of $\left.\mathbf{A}\right|_{N_{i}}$ for $i=1,2$ and the algebras $\left(\left.\mathbf{A}\right|_{N_{1}}\right) /\left(\left.\alpha\right|_{N_{1}}\right)$ and $\left(\left.\mathbf{A}\right|_{N_{2}}\right) /\left(\left.\alpha\right|_{N_{2}}\right)$ are isomorphic. Furthermore these quotient algebras are polynomially equivalent to exactly one algebra (up to isomorphism) from the following list:

(1) a faithful $G$-set, for some finite group $G$,

(2) a vector space,

(3) a two-element Boolean algebra,

(4) a two-element lattice,

(5) a two-element semilattice. 
We say that the type of the prime quotient $\langle\alpha, \beta\rangle$ is equal to $i$ if the algebra $\left(\left.\mathbf{A}\right|_{N_{1}}\right) /\left(\left.\alpha\right|_{N_{1}}\right)$ is polynomially equivalent to an algebra in the ith entry of this list. We denote this type by $\operatorname{typ}(\alpha, \beta)$.

\section{Definition 3.4}

(1) Let $\langle\delta, \gamma\rangle$ be any congruence quotient of a finite algebra $\mathbf{A}$. We define $\operatorname{typ}\{\delta, \gamma\}$ to be the set

$$
\{\operatorname{typ}(\alpha, \beta): \delta \leq \alpha \prec \beta \leq \gamma\} .
$$

(2) For a finite algebra $\mathbf{A}$ we define typ $\{\mathbf{A}\}$ to be $\operatorname{typ}\left\{0_{A}, 1_{A}\right\}$.

(3) For a class $\mathcal{K}$ of algebras we define typ $\{\mathcal{K}\}$ to be the set

$$
\bigcup\{\operatorname{typ}\{\mathbf{A}\}: \mathbf{A} \in \mathcal{K} \text { and } \mathbf{A} \text { is finite }\} \text {. }
$$

We say that a finite algebra omits type $i$ if $i \notin \operatorname{typ}\{\mathbf{A}\}$. A class $\mathcal{K}$ omits type $i$ if every finite member of $\mathcal{K}$ does so.

COROLLARY 3.5 Let A be a finite algebra and let $\alpha$ and $\beta$ be congruences of $\mathbf{A} \cdot \operatorname{typ}\{\alpha, \beta\} \subseteq\{\mathbf{1}, \mathbf{2}\}$ if and only if $\beta$ is solvable over $\alpha$.

One of the interesting aspects of the work of Hobby and McKenzie can be found in chapters 8 and 9 of [9]. There they show that certain Maltsev conditions (for locally finite varieties) are easily expressible in the language of tame congruence theory. For example, a locally finite variety $\mathcal{V}$ is $n$ permutable for some $n$ if and only if $\mathcal{V}$ omits types $\mathbf{1}, \mathbf{4}$ and $\mathbf{5}$.

In chapter 11 of [9] the following theorem is proved.

THEOREM 3.6 Let $\mathcal{V}$ be a locally finite decidable variety. Then $\mathcal{V}$ omits types $\mathbf{4}$ and $\mathbf{5}$.

\section{A Sketch of the Proof}

Throughout this section we fix a decidable locally finite variety $\mathcal{V}$. From Theorem 3.6 we know that $\operatorname{typ}\{\mathcal{V}\} \subseteq\{\mathbf{1}, \mathbf{2}, \mathbf{3}\}$. We first define three subvarieties, $\mathcal{V}_{1}, \mathcal{V}_{2}$ and $\mathcal{V}_{3}$, of $\mathcal{V}$; and we prove that every member of $\mathcal{V}$ is a subdirect product of three algebras belonging to these subvarieties. 


\section{Definition 4.1}

(1) A subdirect product of the algebras $\left\langle\mathbf{B}_{i}: i \in I\right\rangle$ is an algebra $\mathbf{A} \leq \prod_{i \in I} \mathbf{B}_{i}$ such that $\mathbf{A}$ maps onto each of the algebras $\mathbf{B}_{i}$ via the coordinate projection. An embedding $f: A \longrightarrow \prod_{i \in I} \mathbf{B}_{i}$ is called subdirect if $f(\mathbf{A})$ is a subdirect product of the $\mathbf{B}_{i}$ 's.

(2) An irredundant subdirect product of $\left\langle\mathbf{B}_{i}: i \in I\right\rangle$ is a subdirect product $\mathbf{A}$ of $\left\langle\mathbf{B}_{i}: i \in I\right\rangle$ such that for each $i \in I$, A fails to be embedded in $\prod_{j \neq i} \mathbf{B}_{j}$ via the natural projection.

(3) A subdirect product $\mathbf{A}$ of $\left\langle\mathbf{B}_{i}: i \in I\right\rangle$ is called direct iff $\mathbf{A}=\prod_{i \in I} \mathbf{B}_{i}$.

(4) An algebra $\mathbf{A}$ is called subdirectly irreducible if and only if $|A|>1$ and for every subdirect embedding $f: A \longrightarrow \prod_{i \in I} \mathbf{B}_{i}$, there is some $i \in I$ such that the composition of $f$ with the projection onto $\mathbf{B}_{i}$ is an isomorphism between $\mathbf{A}$ and $\mathbf{B}_{i}$.

Equivalent to an algebra $\mathbf{A}$ being subdirectly irreducible is the existence of a smallest nonzero congruence in Con $\mathbf{A}$. We call this congruence (when it exists) the monolith of $\mathbf{A}$.

Definition 4.2 For $1 \leq i \leq 3$, let $\mathcal{S}_{i}$ be the class of all finite, subdirectly irreducible algebras $\mathbf{A} \in \mathcal{V}$ such that the type of $\left\langle 0_{A}, \beta\right\rangle$ is i, where $\beta$ is the monolith of $\mathbf{A}$. We define $\mathcal{V}_{i}$ to be the variety generated by $\mathcal{S}_{i}$.

Here are three easy consequences of the definition.

\section{THEOREM 4.3}

(i) Every finite subdirectly irreducible algebra in $\mathcal{V}$ belongs to $\mathcal{V}_{1} \cup \mathcal{V}_{2} \cup \mathcal{V}_{3}$.

(ii) Every locally solvable algebra in $\mathcal{V}$ belongs to $\mathcal{V}_{1} \vee \mathcal{V}_{2}$

(iii) $\mathcal{V}=\mathcal{V}_{1} \vee \mathcal{V}_{2} \vee \mathcal{V}_{3}$; in fact, every algebra in $\mathcal{V}$ is a subdirect product of three algebras $\mathbf{C}_{1}, \mathbf{C}_{2}$ and $\mathbf{C}_{3}$ belonging, respectively, to $\mathcal{V}_{1}, \mathcal{V}_{2}$ and $\mathcal{V}_{3}$. 
Proof. Since $\mathcal{V}$ is decidable, then it follows from Theorem 3.6 that every finite, subdirectly irreducible algebra in $\mathcal{V}$ belongs to $\mathcal{S}_{1} \cup \mathcal{S}_{2} \cup \mathcal{S}_{3}$.

Let $\mathbf{A} \in \mathcal{V}$ be locally solvable. Since $\mathcal{V}$ is locally finite, every finitely generated subalgebra of $\mathbf{A}$ is finite; and so $\mathbf{A}$ belongs to the variety generated by its finite subalgebras. Hence it suffices to prove that all finite subalgebras of $\mathbf{A}$ belong to $\mathcal{V}_{1} \vee \mathcal{V}_{2}$. Let $\mathbf{B}$ be a finite subalgebra of $\mathbf{A}$. $\mathbf{B}$ is a subdirect product of subdirectly irreducible homomorphic images of $\mathbf{B}$, which are solvable, and hence have monoliths of types $\mathbf{1}$ or $\mathbf{2}$. Thus $\mathbf{B} \in \operatorname{SP}_{\text {fin }}\left(\mathcal{S}_{1} \cup \mathcal{S}_{2}\right)$, implying that $\mathbf{B} \in \mathcal{V}_{1} \vee \mathcal{V}_{2}$.

It follows easily from part (i) that every finite algebra in $\mathcal{V}$ can be embedded into a product $\mathbf{C}_{1} \times \mathbf{C}_{2} \times \mathbf{C}_{3}$ with $\mathbf{C}_{i} \in \mathcal{V}_{i}$. The class of algebras that can be so embedded is closed under the formation of ultraproducts and of subalgebras. The statement thus follows from the fact that every locally finite algebra can be embedded into an ultraproduct of its finite subalgebras.

With the three subvarieties $\mathcal{V}_{1}, \mathcal{V}_{2}$ and $\mathcal{V}_{3}$ defined, we now set out to describe the structure of each of them. The next lemmas are used to show that $\mathcal{V}_{3}$ is a discriminator variety.

LEMMA 4.4 Let $\mathbf{F}$ be a finite, subdirectly irreducible, centerless algebra in $\mathcal{V}$. Every subalgebra of $\mathbf{F}$ having at least two elements is simple and non-Abelian.

LEMMA 4.5 Every irredundant subdirect product of finitely many algebras in $\mathcal{S}_{3}$ is direct.

THEOREM $4.6 \mathcal{V}_{3}$ is a discriminator variety.

Proof. By Lemma 4.4, $\mathcal{V}_{3}$ is generated by the class of finite, simple, non-Abelian algebras in $\mathcal{V}_{3}$. Thus if $\mathcal{V}_{3}$ can be shown to be Maltsev, then it will follow from Theorem 9.1 of [3] that $\mathcal{V}_{3}$ is a discriminator variety.

Let $\mathbf{F}$ be the free algebra on three generators in $\mathcal{V}_{3}$. Thus $\mathcal{V}_{3}$ is Maltsev iff $\mathbf{F}$ has permuting congruences. Since $\mathbf{F}$ is finite, and $\mathcal{V}_{3}=\operatorname{HSP}\left(\mathcal{S}_{3}\right)$ is locally finite, there exists a finite set $\mathcal{K} \subset \mathcal{S}_{3}$ such that $\mathbf{F} \in \operatorname{HSP}(\mathcal{K})$. Thus our task is reduced to proving that $\mathcal{V}^{\prime}=\operatorname{HSP}(\mathcal{K})$ is Maltsev. By Lemma 4.4, we can assume that every nontrivial subalgebra of an algebra in $\mathcal{K}$ belongs to $\mathcal{K}$. Then $\mathrm{SP}_{\text {fin }}(\mathcal{K})$ consists of the trivial one-element algebras and the algebras 
isomorphic to irredundant subdirect products of finitely many members of $\mathcal{K}$. By Lemma 4.5, we have $\mathrm{SP}_{\text {fin }}(\mathcal{K}) \subset \mathrm{P}(\mathcal{K})$. Thus by Theorem 3.4 of [15], $\mathcal{V}^{\prime}$ is Maltsev.

The following lemma and corollary are needed in our investigation of the subvarieties $\mathcal{V}_{1}$ and $\mathcal{V}_{2}$.

LEMMA 4.7 If $\mathbf{A}$ is any finite algebra in $\mathcal{V}$, then $\mathbf{A} / Z(\mathbf{A})$ is a centerless algebra.

COROLLARY 4.8 If $\mathbf{A} \in \mathcal{V}$ then $Z(\mathbf{A})$ is the largest locally solvable congruence of $\mathbf{A}$. Every locally solvable algebra in $\mathcal{V}$ is Abelian.

We now introduce a concept that will allow us to characterize the subvarieties $\mathcal{V}_{1}$ and $\mathcal{V}_{2}$

Definition 4.9 Let $\mathbf{A}$ be a finite algebra. If $\mathbf{i}, \mathbf{j} \in\{\mathbf{1}, \ldots, \mathbf{5}\}$ are distinct types, we say that $\mathbf{A}$ possesses the $(\mathbf{i}, \mathbf{j})$ transfer principle iff for all $\chi_{0}, \chi_{1}, \chi_{2} \in$ Con $\mathbf{A}$, if $\chi_{0} \prec \chi_{1} \prec \chi_{2}$ and $\operatorname{typ}\left(\chi_{0}, \chi_{1}\right)=\mathbf{i}$ and $\operatorname{typ}\left(\chi_{1}, \chi_{2}\right)=\mathbf{j}$, then there exists $\beta \leq \chi_{2}$ such that $\chi_{0} \prec \beta$ and $\operatorname{typ}\left(\chi_{0}, \beta\right)=\mathbf{j}$.

A locally finite variety possesses the $(\mathbf{i}, \mathbf{j})$ transfer principle if every finite member does.

THEOREM 4.10 Let $\mathbf{i}, \mathbf{j}$ be distinct members of $\{\mathbf{1}, \mathbf{2}, \mathbf{3}\}$. Every finite algebra in $\mathcal{V}$ possesses the $(\mathbf{i}, \mathbf{j})$ transfer principle.

THEOREM 4.11 $\mathcal{V}_{1} \vee \mathcal{V}_{2}$ is the class of all Abelian algebras in $\mathcal{V}$.

Proof. By Theorem 4.3, every Abelian algebra in $\mathcal{V}$ belongs to $\mathcal{V}_{1} \vee \mathcal{V}_{2}$. To prove the converse, it will suffice, by Corollary 4.8, to prove that every algebra in $\mathcal{V}_{1} \vee \mathcal{V}_{2}$ is locally solvable. Since $\mathcal{V}_{1} \vee \mathcal{V}_{2}$ is generated by the class $\mathcal{S}_{1} \cup \mathcal{S}_{2}$, it will suffice, by Theorem 2.7, to prove that every algebra in $\mathcal{S}_{1} \cup \mathcal{S}_{2}$ is solvable. Now $\mathcal{S}_{1} \cup \mathcal{S}_{2}$ is just the class of finite subdirectly irreducible algebras in $\mathcal{V}$ with Abelian monolith. So let $\mathbf{A}$ be a finite subdirectly irreducible algebra belonging to $\mathcal{V}$ whose monolith $\beta$ is Abelian. We shall show that $\mathbf{A}$ is solvable.

By Theorem 3.6, we have $\operatorname{typ}\{\mathbf{A}\} \subset\{\mathbf{1}, \mathbf{2}, \mathbf{3}\}$. By Corollary 3.5, $\mathbf{A}$ is solvable iff $\operatorname{typ}\{\mathbf{A}\} \subset\{\mathbf{1}, \mathbf{2}\}$. Now in order to reach a contradiction, assume 
that $\mathbf{3} \in \operatorname{typ}\{\mathbf{A}\}$. Among all the prime quotients $\left\langle\chi_{0}, \chi_{1}\right\rangle$ in Con $\mathbf{A}$ with $\operatorname{typ}\left(\chi_{0}, \chi_{1}\right)=\mathbf{3}$, choose one $\langle\delta, \gamma\rangle$ such that the cardinality of the interval $I\left[0_{A}, \gamma\right]$ is as small as possible. Since $\operatorname{typ}\left(0_{A}, \beta\right) \in\{\mathbf{1}, \mathbf{2}\}$ where $\beta$ is the monolith, we have $\delta \neq 0_{A}$. Now choose any $\xi \in$ Con $\mathbf{A}$ such that $\xi \prec \delta$. By our choice of $\langle\delta, \gamma\rangle$, the type of $\langle\xi, \delta\rangle$ is not $\mathbf{3}$-so it must be $\mathbf{1}$ or $\mathbf{2}$. Now by the $(\mathbf{1}, \mathbf{3})$ or the $(\mathbf{2}, \mathbf{3})$ transfer principle, there exists a congruence $\lambda$ such that $\xi \prec \lambda<\gamma$ and $\operatorname{typ}(\xi, \lambda)=\mathbf{3}$. This contradicts our choice of $\langle\delta, \gamma\rangle$ and ends the proof.

We now focus on the Abelian subvariety $\mathcal{A}=\mathcal{V}_{1} \vee \mathcal{V}_{2}$. Using the transfer principals and some tame congruence theory it is possible to show that $\operatorname{typ}\left\{\mathcal{V}_{1}\right\}=\mathbf{1}$ and $\operatorname{typ}\left\{\mathcal{V}_{2}\right\}=\mathbf{2}$. From this we conclude the following.

THEOREM 4.12 The subvariety $\mathcal{V}_{1}$ is strongly Abelian and the subvariety $\mathcal{V}_{2}$ is affine.

To establish the independence of the three subvarieties $\mathcal{V}_{1}, \mathcal{V}_{2}$ and $\mathcal{V}_{3}$, and hence finish the proof of Theorem 2.14, we found it necessary to first characterize the decidable locally finite strongly Abelian varieties. There is a close correspondence between these varieties and multi-sorted unary varieties.

A $k$-sorted unary algebra $\mathbf{A}$ is a structure of the form

$$
\left\langle A_{1}, A_{2}, \ldots, A_{k} ;\left\{f_{i}: i \in I\right\}\right\rangle
$$

where the $A_{i}$ are nonempty disjoint sets and for each $i \in I, f_{i}$ is a map from $A_{\sigma(i)}$ to $A_{\tau(i)}$ for some $\sigma(i)$ and $\tau(i)$ less than or equal to $k$.

By a derived operation of $\mathbf{A}$ we mean an operation obtained by composing some of the $f_{i}$ or one of the identity functions $i d_{i}: A_{i} \longrightarrow A_{i}$. Given a sequence $g_{1}, \ldots, g_{k}$ of derived operations having their ranges contained in $A_{1}, \ldots, A_{k}$ respectively and a sequence of numbers less than or equal to $k$, $\eta=\left\langle n_{1}, \ldots, n_{k}\right\rangle$, we can define a $k$-ary operation $\left[g_{1}, \ldots, g_{k}, \eta\right]$ on the set $A_{1} \times \cdots \times A_{k}$ as follows:

$$
\left[g_{1}, \ldots, g_{k}, \eta\right]\left(\left\langle a_{1}^{1}, \ldots, a_{1}^{k}\right\rangle, \ldots,\left\langle a_{k}^{1}, \ldots, a_{k}^{k}\right\rangle\right)=\left\langle g_{1}\left(a_{n_{1}}^{\sigma(1)}\right), \ldots, g_{k}\left(a_{n_{k}}^{\sigma(k)}\right)\right\rangle,
$$

where the domain of $g_{i}$ is $A_{\sigma(i)}$. Let $C(\mathbf{A})$ be the clone of operations on the set $A_{1} \times \cdots \times A_{k}$ generated by the set of all such $k$-ary operations.

We call an algebra $\mathbf{B}$ quasi-unary if for some $k$-sorted unary algebra $\mathbf{A}$, $\mathbf{B}$ is isomorphic to an algebra with universe $A_{1} \times \cdots \times A_{k}$ and clone equal 
to $C(\mathbf{A})$. It is not hard to show that $\mathbf{B}$ is strongly Abelian. The first step in our characterization of the decidable locally finite strongly Abelian variety is to prove the following lemma.

LEMMA 4.13 Let $\mathcal{S}$ be a decidable locally finite strongly Abelian variety. Then every algebra in $\mathcal{S}$ is quasi-unary.

A $k$-sorted unary algebra $\mathbf{A}$ is called linear if for all nonconstant derived operations $f$ and $g$ of $\mathbf{A}$ having the same domain, there is some other derived operation $h$ (having the appropriate domain and range) such that $\mathbf{A}$ satisfies the equation $f(x) \approx h g(x)$ or the equation $g(x) \approx h f(x)$. We extend this definition to quasi-unary algebras in the natural way, i.e., a quasi-unary algebra $\mathbf{B}$ is called linear if the multi-sorted unary algebra that is associated with it is linear.

THEOREM 4.14 Let $\mathcal{S}$ be a locally finite strongly Abelian variety of finite type. Then $\mathcal{S}$ is decidable if and only if every algebra in $\mathcal{S}$ is quasi-unary and linear. This is equivalent to having some generating algebra of $\mathcal{S}$ of this form.

\section{Conclusion}

Theorem 2.14 reduces the study of decidable locally finite varieties to the examination of decidable locally finite varieties that fall into one of the three special cases. As we have noted, the decidable locally finite strongly Abelian varieties have been characterized.

For locally finite discriminator varieties, no criterion for decidability is known. We begin our list of open problems with this one.

Problem 1: Which locally finite discriminator varieties are undecidable?

S. Burris and H. Werner [6] proved that every finitely generated discriminator variety of finite type is decidable. Some more recent results on Problem 1 can be found in S. Burris [2] and S. Burris, R. McKenzie and M. Valeriote [4].

The decidability question for locally finite affine varieties is very interesting and seems to be very difficult. Corresponding to a locally finite affine 
variety $\mathcal{V}$ there is a finite ring $\mathbf{R}$ such that, according to Burris and McKenzie [3] Theorem 10.6, the variety of left unitary modules over $\mathbf{R}$ is decidable if and only if $\mathcal{V}$ is decidable. Thus we have the next problem.

Problem 2: Which finite rings $\mathbf{R}$ with unit have the property that the variety of left unitary modules over $\mathbf{R}$ is decidable?

W. Baur [1] constructed some finite rings having an undecidable theory of modules. F. Point [18] and M. Prest [19] have made a deep study of this problem.

The methods used to prove Theorem 2.14 are clearly applicable to the next problems, although new methods may also be needed.

Problem 3: Which locally finite quasivarieties (universal Horn classes) are undecidable?

Problem 4: For which locally finite varieties is the class of finite members undecidable?

In A. P. Zamyatin [24], a list is given of all the varieties of rings whose class of finite members is decidable. Recently P. M. Idziak [10, 11, 12] has characterized those finitely generated congruence distributive varieties of finite type whose class of finite members is decidable. He proves that such a variety must be congruence permutable and the congruence lattice of every subdirectly irreducible algebra in the variety must be linearly ordered. If either of these conditions fail then the variety is shown to be $\omega$-unstructured.

In [22] it is shown that every locally finite, Abelian variety whose theory of its finite members is decidable is the varietal product of a strongly Abelian variety and an affine variety.

We close with a corollary of our structure theory.

COROLLARY 5.1 There exists an algorithm which produces, given a finite algebra of finite type, a finite ring with unit such that the algebra generates a decidable variety iff the variety of left unitary modules over the ring is decidable.

Proof. Due to the restrictive nature of the structure of any finite algebra that generates a decidable variety, such an algorithm can be found. We can use the algorithm described in Burris, McKenzie (Theorem 11.3), adding to it the test for decidability of a strongly Abelian variety contained in our Theorem 4.14 . 


\section{References}

[1] W. Baur. Undecidablility of the theory of Abelian groups with a subgroup. Proc. Amer. Math. Soc., 55:125-128, 1976.

[2] S. Burris. Iterated discriminator varieties have undecidable theories. Algebra Universalis, 21:54-61, 1985.

[3] S. Burris and R. McKenzie. Decidability and Boolean Representations, volume 246 of Memoirs of the American Mathematical Society. American Mathematical Society, 1981.

[4] S. Burris, R. McKenzie, and M. Valeriote. Decidable discriminator varieties from unary varieties. Journal of Symbolic Logic, 56:1355-1368, 1991.

[5] S. Burris and H.P. Sankappanavar. A Course in Universal Algebra. SpringerVerlag, 1981.

[6] S. Burris and H. Werner. Sheaf constructions and their elementary properties. Trans. Amer. Math. Soc., 248:269-309, 1979.

[7] Yu. L. Ershov. Elementary theories of groups. Dokl. Akad. Nauk SSSR, 6:1240-1243, 1972.

[8] R. Freese and R. McKenzie. Commutator Theory for Congruence Modular Varieties, volume 125 of London Mathematical Society Lecture Note Series. Cambridge University Press, 1987.

[9] D. Hobby and R. McKenzie. The Structure of Finite Algebras, volume 76 of Contemporary Mathematics. American Mathematical Society, 1988.

[10] P. Idziak. Reduced sub-powers and the decision problem for finite algebras in arithmetical varieties. Algebra Universalis, 25:365-383, 1988.

[11] P. Idziak. Varieties with decidable finite algebras I: linearity. Algebra Universalis, 26:234-246, 1989.

[12] P. Idziak. Varieties with decidable finite algebras II: Permutability. Algebra Universalis, 26:247-256, 1989.

[13] I. A. Lavrov. Effective inseparability of the sets of identically true formulae and finitely refutable formulae for certain elementary theories. Algebra $i$ Logika Seminar 2, 1:5-18, 1963. 
[14] A. I. Maltsev. On the general theory of algebraic systems. Mat. Sb. (N.S.), $35: 3-20,1954$.

[15] R. McKenzie. Narrowness implies uniformity. Algebra Universalis, 15:67-85, 1982.

[16] R. McKenzie, G. McNulty, and W. Taylor. Algebras, Lattices, Varieties Volume 1. Wadsworth and Brooks/Cole, Monterey, California, 1987.

[17] R. McKenzie and M. Valeriote. The Structure of Locally Finite Decidable Varieties, volume 79 of Progress in Mathematics. Birkhäuser Boston, 1989.

[18] F. Point. Problèmes de décidabilité pour les théories des modules. Bull. Belg. Math. Soc. Ser. B, 38:58-74, 1986.

[19] M. Prest. Model Theory and Modules, volume 130 of London Math. Soc. Lecture Note Series. Cambridge University Press, 1988.

[20] W. Szmielew. Elementary properties of Abelian groups. Fund. Math., 41:203$271,1955$.

[21] A. Tarski. Arithmetical classes and types of Boolean algebras. Bull. Amer. Math. Soc., 55:64, 1949.

[22] M. Valeriote. Abelian varieties having their class of finite members decidable. abstract, 1989.

[23] A. P. Zamyatin. A prevariety of semigroups whose elementary theory is solvable. Algebra and Logic, 12:233-241, 1973.

[24] A. P. Zamyatin. Varieties of associative rings whose elementary theory is decidable. Soviet Math. Dokl., 17:996-999, 1976.

[25] A. P. Zamyatin. A non-Abelian variety of groups has an undecidable elementary theory. Algebra and Logic, 17:13-17, 1978.

Department of Mathematics, University of California, BerkeLEY, CALIFornia 94720

Department of Mathematics and Statistics, McMaster University, Hamilton, Ontario, Canada, L8S 4K1. 